Kragujevac Journal of Mathematics

Volume 38(2) (2014), Pages 229-244.

\title{
ON ZERO SETS AND EMBEDDINGS OF SOME NEW ANALYTIC FUNCTION SPACES IN THE UNIT DISK
}

\author{
ROMI F. SHAMOYAN ${ }^{1}$ AND OLIVERA R. MIHIĆ ${ }^{2}$
}

\begin{abstract}
We introduce and study certain new scales of analytic functions of area Nevanlinna type in the unit disk and solve various problems connected with zero sets and embeddings in these scales of spaces.
\end{abstract}

\section{INTRODUCTION}

Assuming that $\mathbf{D}=\{z \in \mathbb{C}|:| z \mid<1\}$ is the unit disk of the finite complex plane $\mathbb{C}, \mathbf{T}$ is the boundary of $\mathbf{D}, \mathbf{T}=\{z \in \mathbb{C}:|z|=1\}$ and $H(\mathbf{D})$ is the space of all functions holomorphic in $\mathbf{D}$ we introduce the classes of functions

$$
N_{\alpha}^{\infty}(\mathbf{D})=\left\{f \in H(\mathbf{D}): T(r, f) \leq C_{f}(1-r)^{-\alpha}, 0 \leq r<1, \alpha \geq 0\right\}
$$

where $T(r, f)=T_{1}(r, f)$ is the classical and well-known Nevanlinna characteristic defined by $T_{p}^{p}(r, f)=\frac{1}{2 \pi} \int_{\mathbf{T}}\left(\log ^{+}|f(r \xi)|\right)^{p} d \xi$, where $a^{+}=\max \{0, a\}, a \in \mathbb{R}$, (see for example [4], [7]). We remark $\left(\log ^{+}|f|\right)^{p}$ is subharmonic for any $p \geq 1$ and for any analytic function in the unit disk and this fact is crucial for various embeddings between analytic area Navenlinna type spaces which we noted in this paper.

It is obvious that if $\alpha=0$ then $N_{0}^{\infty}=N$, where $N$ is a classical Nevanlinna class. The following statement holds by Nevanlinnas classical result on the parametric representation of $N$ [4], [7]. The $N$ class coincides with the set of functions representable in the form

$$
f(z)=C_{\lambda} z^{\lambda} B\left(z,\left\{z_{k}\right\}\right) \exp \left(\int_{-\pi}^{\pi} \frac{d \mu(\theta)}{1-z e^{-i \theta}}\right), z \in \mathbf{D}
$$

where $C_{\lambda}$ is any complex number, $\lambda$ is any nonnegative integer, $B\left(z,\left\{z_{k}\right\}\right)$ is the classical Blaschke product with zeros $\left\{z_{k}\right\}_{k=1}^{\infty} \subset \mathbf{D}$ enumerated according to their

\footnotetext{
Key words and phrases. Weierstrass - type products, Area Nevanlinna - type spaces, Nevanlinna characteristic, Embeddings, Zero sets.

2010 Mathematics Subject Classification. Primary: 32A10. Secondary: 32A37.

Received: November 28, 2013

Accepted: September 26, 2014.
} 
multiplicities and satisfying the Blaschke density condition $\sum_{k=1}^{\infty}\left(1-\left|z_{k}\right|\right)<\infty$, and $\mu(\theta)$ is any function of bounded variation on $[-\pi, \pi]$. Descriptions of zero sets and complete parametric representations of $N_{\alpha}^{\infty}$ spaces can be seen in [1] and [21].

Also, by $m_{2}(\xi)$ we denote standard normalized Lebesgue area measure.

Everywhere below by $n_{f}(t)=n(t)$ we denote the number of zeros of an analytic function $f$ in the disk $|z| \leq t<1$ and by $Z(X)$ the zero set of an analytic class $X, X \subset H(\mathbf{D})$, i. e.

$$
Z(X)=\left\{\Lambda \subset \mathbf{D}: \exists f \in X \backslash\{0\}, f_{\left.\right|_{\Lambda}}=0\right\}
$$

Also, by $n_{k}$ we denote $n\left(1-2^{-k}\right)$, i. e. $n_{k}=n\left(1-2^{-k}\right), k=1,2, \ldots$

Let

$$
(N A)_{p, q, \gamma, \delta}(\mathbf{D})=\left\{f \in H(\mathbf{D}): \int_{0}^{1}\left[\sup _{0<\tau<R} T_{q}(\tau, f)(1-\tau)^{\gamma}\right]^{p}(1-R)^{\delta} d R<\infty\right\},
$$

where $\gamma \geq 0, \delta>-1,0<p<\infty, 0<q<\infty$.

When $q=1$ then we use $(N A)_{p, \gamma, \delta}(\mathbf{D})$.

The classes we study in the first part of this paper are closely connected with classical weighted analytic Nevanlinna spaces in the unit disk (case $\delta=-1, p=$ $q=1)$. Zero sets and parametric representation of these last classes were obtained recently in [18]. On the other hand putting formally $\delta=-1, p=q=1$ and replacing sup by integration we get well-studied Nevanlinna-Djrbashian spaces (see [4], [14] and references there). This motivates the study of scales of spaces we defined above in this paper.

It is not difficult to see taking $t$ parameter big enough that our spaces are contained in weighted analytic Nevanlinna $N_{t}^{p}(\mathbf{D})$ spaces. On the other hand it is well known that this $N_{t}^{p}(\mathbf{D})$ classes are larger that any Hardy $H^{s}$ and Bergman $A_{\tau}^{s}$, where $s \in$ $(0, \infty), \tau>-1$, where weighted Nevanlinna spaces are defined as follows

$$
N_{t}^{p}(\mathbf{D})=\left\{f \in H(\mathbf{D}): \sup _{r \in(0,1)} T_{p}(f, r)(1-r)^{t}<\infty\right\} .
$$

We start the investigation of $(N A)_{p, \gamma, \delta}(\mathbf{D})$ in [1] and one of the intention of this note is to provide some results from there on zero sets in more general form for $(N A)_{p, q, \gamma, \delta}(\mathbf{D})$ spaces. Let also

$$
\begin{aligned}
& L\left(A_{\gamma}^{p, q}\right)(\mathbf{D})= \\
& =\left\{f \in H(\mathbf{D}):\|f\|_{L\left(A_{\gamma}^{p, q}\right)}=\int_{0}^{1}\left(\int_{-\pi}^{\pi}\left(\ln ^{+}\left|f\left(r e^{i \varphi}\right)\right|\right)^{p} d \varphi\right)^{q / p}(1-r)^{\gamma} d r<\infty\right\},
\end{aligned}
$$

where $0<p<\infty, 0<q<\infty$ and

$$
\begin{aligned}
& L\left(F_{\gamma}^{p, q}\right)(\mathbf{D})= \\
& =\left\{f \in H(\mathbf{D}):\|f\|_{L\left(F_{\gamma}^{p, q}\right)}=\int_{-\pi}^{\pi}\left(\int_{0}^{1}\left(\ln ^{+}\left|f\left(r e^{i \varphi}\right)\right|\right)^{q}(1-r)^{\gamma} d r\right)^{p / q} d \varphi<\infty\right\},
\end{aligned}
$$

where $0<p<\infty, 0<q<\infty, \gamma>-1$. 
Using direct methods or various embeddings, in this paper we will study zero sets of this $(N A)_{p, q, \gamma, \delta}(\mathbf{D}), L\left(A_{\gamma}^{p, q}\right)(\mathbf{D})$ and $L\left(F_{\gamma}^{p, q}\right)(\mathbf{D})$ spaces and as well as other zero sets of similar analytic Nevanlinna type spaces in the unit disk and some bounded domains.

Throughout the paper $C$ sometimes with indices stands for various positive constants which can be different even in a chain of inequalities and are independent of the discussed functions or variables.

In his celebrated solution of the corona problem in the disk, Carleson [2] introduced an important class of measures to study the structure of the Hardy spaces of the unit disk $\mathbf{D} \subset \mathbb{C}$. Let $H(\mathbf{A})$ be a Banach space of holomorphic functions on a domain $\mathbf{A} \subset \mathbb{C}$, and assume that $H(\mathbf{A})$ is contained in $L^{p}(\mathbf{A})$ for some $p>0$. A finite positive Borel measure $\mu$ on $\mathbf{A}$ is a Carleson measure of $H(\mathbf{A})$ if there exists a constant $C>0$ such that for all $f \in H(\mathbf{A})$

$$
\int_{\mathbf{A}}|f|^{p} d \mu \leq C\|f\|_{H(\mathbf{A})}^{p} .
$$

Carleson studied Carleson measures of the Hardy spaces $H^{p}(\mathbf{D})$ showing that a finite positive Borel measure $\mu$ is a Carleson measure of $H^{p}(\mathbf{D})$ if and only if there exists a constant $C>0$ such that $\mu\left(S_{\theta_{0}, h}\right) \leq C h$ for all sets

$$
S_{\theta_{0}, h}=\left\{r e^{i \theta} \in \mathbf{D}: 1-h \leq r<1,\left|\theta-\theta_{0}\right| \leq h\right\},
$$

(see [2]). In particular the set of Carleson measures of $H^{p}(\mathbf{D})$ does not depend on $p$. In 1975, Hastings [6], [11], [12] proved a similar characterization for the Carleson measures of the Bergman spaces $A^{p}(\mathbf{D})$ : a finite positive Borel measure $\mu$ is a Carleson measure of $A^{p}(\mathbf{D})$ if and only if there exists a constant $C>0$ such that $\mu\left(S_{\theta_{0}, h}\right) \leq C h^{2}$ for all $\theta \in[0,2 \pi]$ and $h \in(0,1)$. As a consequence, again, the set of Carleson measures of $A^{p}(\mathbf{D})$ does not depend on $p$.

Let $d m_{2}$ denote the Lebesgue measure on unit disk $\mathbf{D}$ normalized such that $m_{2}(\mathbf{D})=$ 1. Given $\alpha>-1$, let $\nu_{\alpha}$ be the weighted measure defined by $d \nu_{\alpha}(z)=(\alpha+1)(1-$ $\left.|z|^{2}\right)^{\alpha} d m_{2}(z)$. For $0<p<\infty$ and $\alpha>-1$, we let $N_{\alpha}^{p}$ denote the space of all functions $f \in H(\mathbf{D})$ such that

$$
\int_{\mathbf{D}}\left(\log ^{+}|f|\right)^{p} d \nu_{\alpha}<\infty
$$

We will refer to the spaces $N_{\alpha}^{p}$ as (weighted) area Nevanlinna spaces. Obviously, each area Nevanlinna space is a subalgebra of $H(\mathbf{D})$. The area Nevanlinna spaces are large in the sense that each $N_{\alpha}^{p}$ contains all the well-known Bergman spaces. This follows from standard growth estimates for Bergman functions (see [8], Lemma 3.2).

Let again $\mu$ be a positive finite Borel measure on $\mathbf{D}$. We introduce the spaces $N^{p}(\mu), 1 \leq p<\infty$, which we call area Nevanlinna- Lebesgue spaces. We define $N^{p}(\mu)$ to be the space of all measurable functions $h$ on $\mathbf{D}$ such that

$$
\|h\|_{N^{p}(\mu)}^{p}=\int_{\mathbf{D}}(\log (1+|h|))^{p} d \mu<\infty .
$$


Evidently, $N_{\alpha}^{p}(\mathbf{D})$ is a closed subspace of $N^{p}\left(\nu_{\alpha}\right)$. We also recall the well-known (weighted) Bergman spaces $A_{\alpha}^{p}(\mathbf{D})=L^{p}\left(\nu_{\alpha}\right) \cap H(\mathbf{D})$, where $\alpha>-1$ and $1 \leq p<\infty$. Note that while the area Nevanlinna(-Lebesgue) spaces are not Banach spaces, the restriction $1 \leq p<\infty$ means that the spaces $A_{\alpha}^{p}$ are.

Let $\mathcal{J}$ denote the integration operator defined by $\mathcal{J} f(z)=\int_{0}^{z} f(\zeta) d \zeta, z \in \mathbf{D}$ for $f \in H(\mathbf{D})$. Also, let $\mathcal{D}=\frac{\partial}{\partial z}$ denote the differentiation operator.

Theorem 1.1. [3] Let $1 \leq p<\infty$ and $\alpha>-1$. Given $\nu \geq 0$ be positive Borel measure on $\mathbf{D}$, the following conditions are equivalent:

(i) $A_{\alpha}^{p}(\mathbf{D}) \subset L^{p}(\nu)$;

(ii) $N_{\alpha}^{p}(\mathbf{D}) \subset N^{p}(\nu)$;

(iii) The embedding $N_{\alpha}^{p}(\mathbf{D}) \subset N^{p}(\nu)$ is metrically bounded;

(iv) $\mathcal{J} N_{\alpha}^{p}(\mathbf{D}) \subset N^{p}(\nu)$

(v) J : $N_{\alpha}^{p} \rightarrow N^{p}(\nu)$ is metrically bounded;

(vi) $\mathcal{D}\left(N_{\alpha}^{p}(\mathbf{D})\right) \subset N^{p}(\nu)$.

Below we provide embeddings of this type for various new analytic area Nevanlinna type spaces in the unit disk.

\section{Zero sets and embeddings of some analytic area Nevanlinna type SPACES IN THE UNIT DISK}

In this section we, in particular, collect various assertions and facts that will be used by us in main section of the paper. First we introduce a Weierstrass type infinite product (see [7, Chapter 1]) and we give an estimate for it which is crucial for our main results.

Proposition 2.1. [4] Let $\left\{z_{k}\right\}_{k=1}^{\infty}$ be a sequence in the unit disk, $\left\{z_{k}\right\}_{k=1}^{\infty} \subset \mathbf{D}$, satisfying condition $\sum_{k=1}^{\infty}\left(1-\left|z_{k}\right|\right)^{t+2}<\infty, t>-1$. Then for such $t$ and $z \in \mathbf{D}$ the infinite product

$$
\Pi_{t}\left(z,\left\{z_{k}\right\}\right)=\prod_{k=1}^{\infty}\left(1-\frac{z}{z_{k}}\right) \exp \left(\frac{-(t+1)}{\pi} \int_{\mathbf{D}} \frac{\left(1-|\xi|^{2}\right)^{t} \ln \left|1-\frac{\xi}{z_{k}}\right|}{(1-\bar{\xi} z)^{t+2}} d m_{2}(\xi)\right)
$$

converges absolutely and uniformly inside $\mathbf{D}$ where it presents an analytic function with zeros $\left\{z_{k}\right\}_{k=1}^{\infty}$.

Proposition 2.2. [4] If $\left\{z_{k}\right\}_{k=1}^{\infty} \subset \mathbf{D}$, and $\sum_{k=1}^{\infty}\left(1-\left|z_{k}\right|\right)^{t+2}<\infty, t>-1$, then the following estimate holds for $\Pi_{t}\left(z,\left\{z_{k}\right\}\right)$ product

$$
\ln ^{+}\left|\Pi_{t}\left(z,\left\{z_{k}\right\}\right)\right| \leq C_{t} \sum_{k=1}^{\infty} \frac{\left(1-\left|z_{k}\right|^{2}\right)^{t+2}}{\left|1-z \bar{z}_{k}\right|^{\mid t+2}}, z \in \mathbf{D}
$$

where $C_{t}>0$ is a constant depending solely on $t$.

We remind that when $q=1$ we remove it from list of indexes and use the following notation $(N A)_{p, \gamma, \delta}(\mathbf{D})$ instead of $(N A)_{p, 1, \gamma, \delta}(\mathbf{D})$. 
Theorem 2.1. Let $0<p<\infty, \gamma \geq 0, \delta \geq 0$. If $\left\{z_{k}\right\}$ is in a zero set of $(N A)_{p, q, \gamma, \delta}(\mathbf{D})$ and $n_{k}=n_{f}\left(1-2^{-k}\right), k=1,2, \ldots, f \in(N A)_{p, q, \gamma, \delta}(\mathbf{D})$, then

$$
\sum_{k=1}^{\infty} \frac{n_{k}^{p}}{2^{k((\gamma+1) p+\delta+1)}}<\infty
$$

holds for $q \geq 1$ and if (2.2) holds, then $\left\{z_{k}\right\}$ is in a zero set of $(N A)_{p, q, \gamma, \delta}(\mathbf{D}), q \leq 1$. If (2.2) is true then $\Pi_{t}\left(z,\left\{z_{k}\right\}\right) \in(N A)_{p, q, \gamma, \delta}(\mathbf{D}), q \leq 1$, for $p \leq 1, t>\frac{\delta+1}{p}+\gamma-1$, and for $p>1, t>\frac{\delta}{p}+\gamma$.

Proof of Theorem 2.1. We assume that $f(0)=1$. By the classical Jensen inequality

$$
\int_{0}^{1}\left[\sup _{0<\tau<R}\left(\int_{0}^{\tau} \frac{n(u)}{u} d u\right)(1-\tau)^{\gamma}\right]^{p}(1-R)^{\delta} d R \leq C\|f\|_{(N A)_{p, q, \gamma, \delta}(\mathbf{D})}^{p}, q \geq 1 .
$$

Therefore, the following inequalities are true for any $R, \widetilde{R} \in\left(\frac{1}{3}, 1\right)$ such that $\widetilde{R}=\frac{3 R-1}{2}<R$ :

$$
\begin{aligned}
\sup _{0<\tau<R} \int_{0}^{\tau} \frac{n(u)}{u} d u(1-\tau)^{\gamma} & \geq C \sup _{\frac{1}{3}<\tau<R} \int_{\tau-\frac{1-\tau}{2}}^{\tau} \frac{n(u)}{u} d u(1-\tau)^{\gamma} \\
& \geq C \sup _{\frac{1}{3}<\tau<R} n\left(\frac{3 \tau-1}{2}\right)(1-\tau)(1-\tau)^{\gamma} \\
& \geq C \sup _{\rho \in(0, \widetilde{R})} n(\rho)(1-\rho)^{\gamma+1} \\
& \geq C \sup _{\rho \in(C, \widetilde{R})} n(\rho)(1-\rho)^{\gamma+1} .
\end{aligned}
$$

Besides, one can see that for $\widetilde{\widetilde{R}}=\widetilde{R}-\frac{1-\widetilde{R}}{2}, \tau_{0}<1$

$$
\|f\|_{(N A)_{p, q, \gamma, \delta}(\mathbf{D})}^{p} \geq C \int_{\tau_{0}}^{1}(1-R)^{\delta} \sup _{\rho \in(\widetilde{R}, \widetilde{R})} n(\rho)^{p}(1-\rho)^{(\gamma+1) p} d R
$$

and

$$
\begin{aligned}
\|f\|_{(N A)_{p, q, \gamma, \delta}(\mathbf{D})}^{p} & \geq C \int_{\tau_{0}}^{1}(1-\widetilde{R})^{(\gamma+1) p+\delta} n(\widetilde{R})^{p} d R \\
& \geq C \int_{\tau_{0}}^{1} n(\widetilde{R})^{p}(1-\widetilde{R})^{(\gamma+1) p+\delta} d \widetilde{R} \\
& \geq C \sum_{k=1}^{\infty} \frac{n_{k}^{p}}{2^{k((\gamma+1) p+\delta+1)}} .
\end{aligned}
$$

For providing the converse statement, we fix a number $t$ assuming that Proposition 2.1 and Proposition 2.2 can be used. Then, we observe that

$$
\|f\|_{(N A)_{p, q, \gamma, \delta}(\mathbf{D})}^{p}=\int_{0}^{1}\left[\sup _{0<\tau<R} T_{q}(\tau, f)(1-\tau)^{\gamma}\right]^{p}(1-R)^{\delta} d R, q \in(0, \infty)
$$


and

$$
\int_{-\pi}^{\pi}|\ln | \Pi_{t}\left(z,\left\{z_{k}\right\}\right)|| d \varphi \leq C \sum_{k=1}^{\infty}\left(1-\left|z_{k}\right|\right)^{t+2} \int_{-\pi}^{\pi} \frac{d \varphi}{\left|1-\tau \tau_{k} e^{i \varphi}\right| t+2}, z=\tau e^{i \varphi}
$$

where it is denoted $\left|z_{k}\right|=\tau_{k}$ and $z_{k}=\tau_{k} \xi_{k}, \tau_{k}=1-\frac{1}{2^{k+1}}$. Hence for $p \leq 1, q \leq 1$

$$
\begin{aligned}
\left\|\Pi_{t}\right\|_{(N A)_{p, q, \gamma, \delta}(\mathbf{D})}^{p} & \leq C \int_{0}^{1}\left[\sum_{k=1}^{\infty} \frac{\left(1-\left|z_{k}\right|\right)^{t+2}}{\left(1-R\left|z_{k}\right|\right)^{t+1-\gamma}}\right]^{p}(1-R)^{\delta} d R \\
& \leq C \int_{0}^{1}(1-R)^{\delta}\left[\int_{0}^{1} \frac{(1-s)^{t+1} n(s) d s}{(1-R s)^{t+1-\gamma}}\right]^{p} d R \\
& \leq C \int_{0}^{1}(1-R)^{\delta} \sum_{k=1}^{\infty} \frac{n_{k}^{p} 2^{-k[(t+1) p+p]}}{\left[1-\left(1-\frac{1}{2^{k+1}}\right) R\right]^{(t+1-\gamma) p}} d R \\
& \leq C \sum_{k=1}^{\infty} n_{k}^{p} \frac{2^{-k(t+1) p} 2^{-k p}}{2^{-k[(t+1-\gamma) p-\delta-1]}} \\
& \leq C \sum_{k=1}^{\infty} \frac{n_{k}^{p}}{2^{k((\gamma+1) p+\delta+1)}},
\end{aligned}
$$

for $t>\frac{\delta+1}{p}+\gamma-1$, since one can easily verify that

$$
\begin{aligned}
& \sum_{k=1}^{\infty} \frac{\left(1-\left|z_{k}\right|\right)^{t+2}}{\left(1-R\left|z_{k}\right|\right)^{t+1-\gamma}}=\int_{0}^{1} \frac{(1-s)^{t+2} d n(s)}{(1-R s)^{t+1-\gamma}} \\
& =\left.\frac{(1-s)^{t+2} n(s)}{(1-R s)^{t+1-\gamma}}\right|_{0} ^{1}-\int_{0}^{1} n(s)\left(\frac{(1-s)^{t+2}}{(1-R s)^{t+1-\gamma}}\right)^{\prime} d s \\
& =-\int_{0}^{1} n(s)\left[\frac{-(t+2)(1-s)^{t+1}}{(1-R s)^{t+1-\gamma}}+\frac{(1-s)^{t+2}(-(t+1-\gamma))}{(1-R s)^{t+2-\gamma}}(-R)\right] d s \\
& =\int_{0}^{1} \frac{n(s)(t+2)(1-s)^{t+1} d s}{(1-R s)^{t+1-\gamma}}-\int_{0}^{1} \frac{n(s)(1-s)^{t+2}}{(1-R s)^{t+2-\gamma}}(t+1-\gamma) R d s \\
& \leq C \int_{0}^{1} \frac{n(s)(1-s)^{t+1}}{(1-R s)^{t+1-\gamma}}(t+2) d s,
\end{aligned}
$$

that

$$
\begin{aligned}
{\left[\int_{0}^{1} \frac{n(s)(1-s)^{t+1}}{(1-R s)^{t+1-\gamma}} d s\right]^{p} } & \leq C\left[\sum_{k=1}^{\infty} \frac{n\left(1-2^{-k-1}\right) 2^{-k(t+1)} 2^{-k}}{\left(1-\rho_{k} R\right)^{t+1-\gamma}}\right]^{p} \\
& \leq C \sum_{k=1}^{\infty} \frac{n_{k}^{p} 2^{-k(t+1) p} 2^{-k p}}{\left(1-\rho_{k} R\right)^{(t+1-\gamma) p}}
\end{aligned}
$$

and that for any $\tau=(t+1-\gamma) p-(\delta+1)>0$ and $\delta>-1$ 


$$
\int_{0}^{1} \frac{(1-R)^{\delta}}{\left(1-\rho_{k} R\right)^{(t+1-\gamma) p}} d R \leq C\left(\frac{1}{2^{-k}}\right)^{\tau}, \rho_{k}=1-\frac{1}{2^{k}} \quad(k=0,1,2, \ldots) .
$$

Now, let $p>1$. Then for the conjugate index $q>1$ deduced by $\frac{1}{p}+\frac{1}{q}=1$ and any $t>\gamma-1$

$$
\begin{aligned}
\left\|\Pi_{t}\right\|_{(N A)_{p, q, \gamma, \delta}(\mathbf{D})}^{p} & \leq C \int_{0}^{1}\left[\int_{0}^{1} \frac{n(s)(1-s)^{t+1} d s}{(1-R s)^{t+1-\gamma}}\right]^{p}(1-R)^{\delta} d R \\
& =C\left[I_{1}+I_{2}\right] \\
& =C \int_{0}^{1}(1-R)^{\frac{\delta}{p}} \psi(R)\left(\int_{0}^{1} \frac{n(s)(1-s)^{t+1} d s}{(1-R s)^{t+1-\gamma}}\right) d R,
\end{aligned}
$$

where is $\psi$ a nonnegative function such that $\|\psi\|_{L^{q}}=1$,

$$
\begin{aligned}
& I_{1}=\int_{0}^{1} \frac{n(s)(1-s)^{t+1}}{(1-R s)^{t+1-\gamma}}\left(\int_{0}^{s} \psi(R)(1-R)^{\frac{\delta}{p}} d R\right) d s \\
& I_{2}=\int_{0}^{1} \frac{n(s)(1-s)^{t+1}}{(1-R s)^{t+1-\gamma}}\left(\int_{s}^{1} \psi(R)(1-R)^{\frac{\delta}{p}} d R\right) d s
\end{aligned}
$$

and

$$
I_{1} \leq C \int_{0}^{1} n(s)(1-s)^{t+1} \int_{0}^{s} \frac{\psi(R)(1-R)^{\frac{\delta}{p}}}{(1-R)^{t+1-\gamma}} d R d s .
$$

Further, by Hardy and Hölder inequalities

$$
\begin{aligned}
I_{1} & \leq C \int_{0}^{1} \frac{n(s)(1-s)^{t+1}}{(1-s)^{t-\gamma-\frac{\delta}{p}}} \int_{0}^{s} \frac{\psi(R)}{(1-R)} d R d s \\
& \leq C\left(\int_{0}^{1}[n(s)]^{p}(1-s)^{\gamma p+p+\delta} d s\right)^{\frac{1}{p}} \\
& \asymp\left(\sum_{k=1}^{\infty} \frac{n_{k}^{p}}{2^{k((\gamma+1) p+\delta+1)}}\right)^{\frac{1}{p}},
\end{aligned}
$$

for $t>\gamma+\frac{\delta}{p}, n_{k}=n_{f}\left(1-2^{-k}\right), k=1,2, \ldots, f \in(N A)_{p, q, \gamma, \delta}(\mathbf{D})$. 
Besides, again by Hölder and Hardy inequalities, for $t>\gamma-1$

$$
\begin{aligned}
I_{2} & =\int_{0}^{1} n(s)(1-s)^{t+1} d s \int_{s}^{1} \frac{(1-R)^{\frac{\delta}{p}} \psi(R)}{(1-R s)^{t+1-\gamma}} d R \\
& \leq C \int_{0}^{1} n(s) \frac{(1-s)^{t+1}}{(1-s)^{t+1}}\left(\int_{s}^{1} \frac{(1-R)^{\frac{\delta}{p}} \psi(R)}{(1-R s)^{-\gamma}} d R\right) d s \\
& =\int_{0}^{1} n(s)\left(\int_{s}^{1} \frac{(1-R)^{\frac{\delta}{p}} \psi(R)}{(1-s)^{-\gamma}} d R\right) d s \\
& \leq C \int_{0}^{1} n(s)(1-s)^{\frac{\delta}{p}+\gamma} \int_{0}^{1-s} \psi(1-u) d u d s \\
& \leq C\left[\int_{0}^{1}(n(s))^{p}(1-s)^{\delta+\gamma p+p} d s\right]^{\frac{1}{p}}\left[\int_{0}^{1}\left(\frac{1}{1-s} \int_{0}^{1-s} \psi(1-u) d u\right)^{q} d s\right]^{\frac{1}{q}} \\
& \leq C\|\psi\|_{L^{q}}\left[\int_{0}^{1}(n(s))^{p}(1-s)^{\delta+\gamma p+p} d s\right]^{\frac{1}{p}} \\
& \asymp C\|\psi\|_{L^{q}}\left(\sum_{k=1}^{\infty} \frac{n_{k}^{p}}{2^{k((\gamma+1) p+\delta+1)}}\right)^{\frac{1}{p}} .
\end{aligned}
$$

The last equivalence relation follows from dyadic decomposition of $(0,1)$ to dyadic intervals like $\left[1-2^{-k}, 1-2^{-(k+1)}\right], k \in \mathbb{N}$. As at the end of proof of Theorem 2.1, it remains to show that the infinite product $\Pi_{t}$ converges for the considered values of $t$. Namely that if $(2.2)$ holds then $\sum_{k=1}^{\infty}\left(1-\left|z_{k}\right|\right)^{t+2}<\infty$. This can be done easily and we omit the details.

Remark 2.1. As we see, using obvious connection between two Nevanlinna characteristics $T_{1}(r, f)$ and $T_{q}(r, f)$ we can extend both assertions of Theorem from [1] partially to all $(N A)_{p, q, \gamma, \delta}(\mathbf{D})$.

Remark 2.2. It will be interesting to know if conditions on zero sets for general $(N A)_{p, q, \gamma, \delta}(\mathbf{D})$ we put above are sharp or not.

Corollary 2.1. [1] Let $0<p<\infty, \gamma \geq 0, \delta \geq 0$. Then the following two statements are equivalent:

(i) $\left\{z_{k}\right\} \in Z\left((N A)_{p, \gamma, \delta}(\mathbf{D})\right)$;

(ii) $\sum_{k=1}^{\infty} \frac{n_{k}^{p}}{2^{k((\gamma+1) p+\delta+1)}}<\infty$.

We add more assertions on zero sets of analytic area Nevanlinna spaces. Using classical Jensen equality we have

$$
\int_{0}^{r} \frac{n(t)}{t} d t=\frac{1}{2 \pi}\left(\int_{-\pi}^{\pi} \ln \left|f\left(r e^{i \varphi}\right)\right| d \varphi\right) .
$$

(We put $f(0)=1$ which is possible.) 
Then from equality above we have

$$
\int_{0}^{r} \frac{n(t)}{t} d t \leq \frac{1}{2 \pi} \int_{-\pi}^{\pi} \ln ^{+}\left|f\left(r e^{i \varphi}\right)\right| d \varphi
$$

Hence for $\alpha>-1$ using the assumption that $g=\left(\log ^{+}|f(z)|\right)^{p}$ is subharmonic for $p \leq 1$ we have [5], [19]

$$
\left(\int_{\mathbf{D}}|g(z)|(1-|z|)^{\alpha} d m_{2}(z)\right)^{p} \leq C \int_{\mathbf{D}}|g(z)|^{p}(1-|z|)^{\alpha p+2 p-2} d m_{2}(z),
$$

where $p \leq 1,(\alpha+2) p>1$.

So we have

$$
\begin{aligned}
J & =\int_{0}^{1}(1-r)^{\alpha} \int_{0}^{r} \frac{n(t)}{t} r d r d t \\
& \left.\leq C \int_{0}^{1}\left(\int_{-\pi}^{\pi}(1-r)^{\alpha p+2 p-2}\left(\ln ^{+}\left|f\left(r e^{i \varphi}\right)\right|\right)^{p} r d r d \varphi\right)^{1 / p}\right)=M_{p} .
\end{aligned}
$$

It is known [1] that

$$
J \geq \sum_{k=1}^{\infty}\left(1-\left|z_{k}\right|\right)^{\alpha+2} .
$$

But [19]

$$
M_{1} \leq C \int_{0}^{1} \int_{-\pi}^{\pi}\left(\ln ^{+}\left|f\left(r e^{i \varphi}\right)\right| d \varphi\right)^{q}(1-r)^{\gamma} d r=C\|f\|_{L\left(A_{\gamma}^{1, q}\right)(\mathbf{D})}, q \leq 1,
$$

for $\gamma=q(\alpha+1)-1$, and

$$
M_{1} \leq C \int_{-\pi}^{\pi}\left(\int_{0}^{1} \ln ^{+}\left|f\left(r e^{i \varphi}\right)\right|(1-r)^{\gamma} d r\right)^{p} d \varphi=C\|f\|_{L\left(F_{\gamma}^{p, 1}\right)(\mathbf{D})}, p \leq 1,
$$

for $\gamma=\alpha+1-\frac{1}{p}$.

Thus we generalized a classical result to mixed norm analytic classes $L\left(A_{\alpha}^{p, q}\right)(\mathbf{D})$ and $L\left(F_{\alpha}^{p, q}\right)(\mathbf{D})$ of area Nevanlinna type for some $\gamma$ depending on $p, q$ and $\alpha$.

Theorem 2.2. If $f \in L\left(A_{\gamma}^{1, q}\right)(\mathbf{D})$ and if $\left(\log ^{+}|f|\right)^{q}$ is subharmonic, $\gamma=q(\alpha+1)-1$, $\gamma>-1, q \leq 1, \alpha>-1$, then if the $\left\{z_{k}\right\}_{k=1}^{\infty}$ is a zero set of $f$ then we have $\sum_{k=1}^{\infty}\left(1-\left|z_{k}\right|\right)^{\alpha+2}<+\infty$.

Similarly, if $f \in L\left(F_{\gamma}^{p, 1}\right)(\mathbf{D})$ and if $\left(\log ^{+}|f|\right)^{p}$ is subharmonic, $\gamma=\alpha-\frac{1}{p}+1$, $\gamma>-1, p \leq 1, \alpha>-1$, then if the $\left\{z_{k}\right\}_{k=1}^{\infty}$ is a zero set of $f$ then we also have $\sum_{k=1}^{\infty}\left(1-\left|z_{k}\right|\right)^{\alpha+2}<+\infty$.

Remark 2.3. For $p=q=1$ this result is a classical [4].

Let $M(r, f)=\max _{|z| \leq r<1}|f(z)|, r \in(0,1], \ln ^{+} x=\max _{x>0}(\ln x, 0)$ and

$$
B_{\alpha, \psi}(\mathbf{D})=\left\{f \in H(\mathbf{D}): \int_{0}^{1}(\ln M(r, f))(1-r)^{\alpha}(\psi(1-r)) d r<+\infty\right\},
$$


where $\alpha>-1, \psi(t)=\exp \left\{-\int_{t}^{1} \frac{\varepsilon(u)}{u} d u\right\}$ and $\varepsilon(t)$ bounded measurable function on $(0,1)$.

Theorem 2.3. [17] Let $\left\{z_{k}\right\}_{1}^{\infty}$ be any sequence from Stolz angle $S_{\delta}$. Let $\sup _{0 \leqslant u<\delta} \frac{|\varepsilon(u)|}{\alpha+1} \leq q<1$ for all $0<\delta<\delta_{0}$, for some $\delta_{0}>0$. Then there is an $f$ function $f \in B_{\alpha, \psi}(\mathbf{D}), f\left(z_{n}\right)=0, f(z) \neq 0$ for $z \neq z_{n}, n=1, \ldots$, if only

$$
\sum_{n=1}^{\infty}\left(1-\left|z_{n}\right|\right)^{\alpha+1}\left(\psi\left(1-\left|z_{n}\right|\right)\right)=M_{\alpha, \psi}<\infty .
$$

We replace space and get similar assertion below. Proof of the theorem that follows is similar to the proof of Theorem 2.3.

Theorem 2.4. Let $\alpha, \beta \geq 0$. Let $\sup _{0 \leqslant u<\delta} \frac{|\varepsilon(u)|}{\alpha+1} \leq q<1$ for all $0<\delta<\delta_{0}$, for some $\delta_{0}>0$. Let

$$
B_{\alpha, \psi, \beta}^{1}(\mathbf{D})=\left\{f \in H(\mathbf{D}): \int_{0}^{1}\left(\sup _{0<r<R}(\ln M(r, f))(1-r)^{\alpha} \psi(1-r)(1-R)^{\beta} d R<\infty\right\} .\right.
$$

Let

$$
B_{\alpha, \psi, \beta}^{2}(\mathbf{D})=\left\{f \in H(\mathbf{D}): \int_{0}^{1}\left(\int_{0}^{R}(\ln M(r, f))(1-r)^{\alpha} \psi(1-r) d r\right)(1-R)^{\beta} d R<\infty\right\} .
$$

Let $\left\{z_{k}\right\}_{1}^{\infty}$ be any sequence from Stolz angle $S_{\delta}$. Then there is a function $f \in$ $B_{\alpha, \psi, \beta}^{1}(\mathbf{D})\left(f \in B_{\alpha, \psi, \beta}^{2}(\mathbf{D})\right), f\left(z_{n}\right)=0, f(z) \neq 0$ for $z \neq z_{n}, n=1, \ldots$, if only

$$
\begin{gathered}
\sum_{n=1}^{\infty}\left(1-\left|z_{n}\right|\right)^{\alpha+\beta+2}\left(\psi\left(1-\left|z_{n}\right|\right)\right)=M_{\alpha+\beta+1, \psi}<\infty, \\
\left(\sum_{n=1}^{\infty}\left(1-\left|z_{n}\right|\right)^{\alpha+\beta+3}\left(\psi\left(1-\left|z_{n}\right|\right)\right)=M_{\alpha+\beta+2, \psi}<\infty\right) .
\end{gathered}
$$

Proof. We sketch the proof of theorem following [17]. Let

$$
\Pi_{p}\left(z,\left\{z_{n}\right\}\right)=\prod_{n=1}^{\infty}\left(1-\frac{1-\left|z_{n}\right|^{2}}{1-\overline{z_{n}} z}\right) \exp \left(\sum_{j=1}^{2 p-1} \frac{1}{j}\left(\frac{1-\left|z_{n}\right|^{2}}{1-\overline{z_{n}} z}\right)^{j}\right) .
$$

Note $\Pi_{p}\left(z,\left\{z_{n}\right\}\right)=0$ only on $z_{n}, n=1,2, \ldots$ and converges uniformly if and only if $\sum_{k=1}^{\infty}\left(1-\left|z_{n}\right|\right)^{2 p}<\infty$. Then [17]

$$
\left.\ln M\left(r, \Pi_{p}\right)\right) \leq \widetilde{C}\left(\sum_{n=1}^{\infty} \frac{\left(1-r_{n}\right)^{2 p}}{\left(1-r_{n} r\right)^{2 p}}\right), r \in(0,1) .
$$


From here we get after some calculations for certain values of $p$ depending on $\alpha$ and $\beta$ following [17]

$$
\begin{aligned}
\left\|\Pi_{p}\right\|_{B_{\alpha, \beta, \psi}^{1}} & \leq C(1) M_{\alpha+\beta+1, \psi}, \\
\left\|\Pi_{p}\right\|_{B_{\alpha, \beta, \psi}^{2}} & \leq C(2) M_{\alpha+\beta+2, \psi},
\end{aligned}
$$

where $\alpha \geq 0, \beta \geq 0$.

We omit easy details refereing the reader to [17].

Finally, we will present some remarks on spaces which we studied before in [20], for $\widetilde{\beta} \geqslant 0, \alpha>-1, \beta>-1,0<p<\infty$.

Let

$$
\widetilde{N}_{\alpha, \beta}^{p}(\mathbf{D})=\left\{f \in H(\mathbf{D}): \int_{0}^{1}\left(\int_{|z| \leq r} \log ^{+}|f(z)|(1-|z|)^{\alpha} d m_{2}(z)\right)^{p}(1-r)^{\beta} d r<\infty\right\}
$$

and $N_{\alpha, \widetilde{\beta}}^{1, \infty}(\mathbf{D})=\left\{f \in H(\mathbf{D}): \sup _{R<1} \int_{|z| \leq R} \log ^{+}|f(z)|(1-|z|)^{\alpha} d m_{2}(z)(1-R)^{\widetilde{\beta}}<\infty\right\}$. Let $S$ be a class of all positive measurable functions on $[0,1]$, so that for some $m_{w}$, $M_{w}, q_{w}, m_{w}, q_{w} \in(0,1]$ and $m_{w} \leq \frac{w(\lambda x)}{w(x)} \leq M_{w}$ for all $x \in(0,1), \lambda \in\left[q_{w}, 1\right]$.

Let $A_{w}^{1}(\mathbf{D})=\left\{f \in H(\mathbf{D}): \int_{\mathbf{D}} \log ^{+}|f(\xi)| w(1-|\xi|) d m_{2}(\xi)<\infty\right\}, w \in S$.

$A_{w}^{1}(\mathbf{D})$ class is well-studied (zero sets, parametric representation), see [17] and various references there. The norm of this space is given by integral in definition of this space.

To have more information about $N_{\alpha, \beta}^{1, \infty}(\mathbf{D}), \widetilde{N}_{\alpha, \beta}^{p}(\mathbf{D})$ we connect them with $A_{w}^{1}(\mathbf{D})$. We define $N_{\alpha, \beta}^{p, \infty}(\mathbf{D})$ replacing $T(r, f)$ by $T_{p}(r, f)$ in definition above of $N_{\alpha, \beta}^{1, \infty}(\mathbf{D})$ space.

Let $\alpha_{w}=\left(\frac{\ln \frac{1}{M}}{\ln \frac{1}{q}}\right), \beta_{w}=\frac{\ln \frac{1}{m}}{\ln \frac{1}{q}}$.

Theorem 2.5. (i) $A_{w}^{1}(\mathbf{D}) \subset N_{\alpha, \beta}^{p, \infty}(\mathbf{D}), w \in S, 0<p<\infty$.

(ii) $A_{w}^{1}(\mathbf{D}) \subset \widetilde{N}_{\alpha, \beta}^{p}(\mathbf{D}), w \in S, 0<p<\infty$.

For following indexes

(i) $\begin{cases}\text { if } & \alpha+\left(\alpha-\alpha_{w}\right)>1, \beta>-1,0<p<\infty \text { or } \\ \text { if } & \beta+\left(\alpha-\alpha_{w}-1\right) p>-1,0<p<\infty .\end{cases}$

(ii) $\quad \begin{cases}\text { if } & \widetilde{\alpha}-p\left(2+\alpha_{w}\right)>-1, \widetilde{\beta} \geq 0,0<p<\infty \text { or } \\ \text { if } & \widetilde{\beta}-p\left(2+\alpha_{w}\right)+\widetilde{\alpha}>-1, \widetilde{\alpha}-p\left(2+\alpha_{w}\right)<-1 .\end{cases}$

Proof of Theorem 2.5. Let $f \in A_{w}^{1}(\mathbf{D})$. From subharmonicity of $\log ^{+}|f(z)|$ we have the following estimate [17]

$$
\log ^{+}|f(z)| \leq C(w) \frac{\|f\|_{w, 1}}{(1-|z|)^{2+\alpha_{w}}}, z \in \mathbf{D} .
$$


This follows similarly as in standard Bergman class. Hence we have integrating by polar coordinates

$$
\begin{aligned}
& \int_{0}^{1}\left(\int_{|z| \leq R} \log ^{+}|f(z)|(1-|z|)^{\alpha} d m_{2}(z)\right)^{p}(1-R)^{\beta} d R \leq C_{w}\|f\|_{w, 1} \cdot I \\
& =C\|f\|_{w, 1} \int_{0}^{1}\left(\int_{|z| \leq R}(1-|z|)^{-\left(2+\alpha_{w}\right)+\alpha} d m_{2}(z)\right)^{p} \times(1-R)^{\beta} d R
\end{aligned}
$$

and

$$
\sup _{R<1} \int_{0}^{R}\left(\int_{\mathbf{T}} \log ^{+}|f(z)| d \xi\right)^{p}(1-|z|)^{\widetilde{\alpha}} d|z|(1-R)^{\widetilde{\beta}} \leq C_{w}\|f\|_{w, 1} \cdot J .
$$

Then we have the following estimates which are easy to check

$$
\begin{aligned}
I & \leq C_{w} \begin{cases}\text { if } & \alpha-\alpha_{w}>1, \beta>-1,0<p<\infty \text { or } \\
\text { if } & \beta+\left(\alpha-\alpha_{w}-1\right) p>-1, \alpha-\alpha_{w}<-1,0<p<\infty .\end{cases} \\
J & \leq \sup _{R<1} \int_{0}^{R}(1-|z|)^{p-2+\alpha_{w}}(1-|z|)^{\widetilde{\alpha}} d|z|(1-R)^{\widetilde{\beta}} \\
& \leq C_{w} \begin{cases}\text { if } & \widetilde{\alpha}-p\left(2+\alpha_{w}\right)>-1, \widetilde{\beta} \geq 0,0<p<\infty \text { or } \\
\text { if } & \widetilde{\beta}-p\left(2+\alpha_{w}\right)+\widetilde{\alpha}>-1, \widetilde{\alpha}-p\left(2+\alpha_{w}\right)<-1 .\end{cases}
\end{aligned}
$$

Theorem 2.5 is proved.

Remark 2.4. Previous assertion has as corollary various assertions on $\widetilde{N}_{\alpha, \beta}^{p}(\mathbf{D}), N_{\alpha, \beta}^{p, \infty}(\mathbf{D})$ based on known results about $A_{w}^{1}(\mathbf{D})$, see [20].

Remark 2.5. Similarly we can find conditions for embedding $A_{w}^{1}(\mathbf{D}) \subset N_{p, q, \gamma, \alpha}(\mathbf{D})$ and we leave this to readers.

Proofs of Proposition 2.3 and Proposition 2.4 as it follows from [10] and [19] are based on arguments from [10] and [19] and their are valid for subharmonic function $\left(\log ^{+}|f(z)|\right)^{s}$ for any $s \geq 1$.

Proposition 2.3. Let $p \geq 1, q \in(0, \infty), \tau=\beta+\frac{q}{p}(\alpha+1)$ then

$$
\int_{0}^{1}\left(\int_{|z|<R}\left(\log ^{+}|f(z)|\right)^{p}(1-r)^{\alpha} d m_{2}(z)\right)^{q / p}(1-R)^{\beta} d r<\infty
$$

if and only if $\int_{0}^{1}\left(\int_{\mathbf{T}}\left(\log ^{+}|f(r \xi)|\right)^{p} d \xi\right)^{q / p}(1-r)^{\tau} d r<\infty$.

Proposition 2.4. Let $1 \leq \min (p, q) \leq s, s \geq 1$ and $\gamma>-1$. Then we have

$$
\left(\int_{\mathbf{D}}\left(\log ^{+}|f(w)|\right)^{s}(1-|w|)^{s(\gamma+1) / q+s / p-2} d m_{2}(w)\right)^{1 / s} \leq C\|f\|_{L\left(A_{\gamma}^{p, q}\right)}, f \in L\left(A_{\gamma}^{p, q}\right)(\mathbf{D}),
$$


and

$$
\left(\int_{\mathbf{D}}\left(\log ^{+}|f(w)|\right)^{s}(1-|w|)^{s(\gamma+1) / q+s / p-2} d m_{2}(w)\right)^{1 / s} \leq C\|f\|_{L\left(F_{\gamma}^{p, q}\right)}, f \in L\left(F_{\gamma}^{p, q}\right)(\mathbf{D}) .
$$

Proposition 2.5. Let $q \geq 1$ and $p \leq s$. Then

$$
\left(\int_{0}^{1} T_{q}^{s}(r, f)(1-|z|)^{\alpha} d|z|\right)^{p / s} \leq \int_{0}^{1}(1-|z|)^{\tau}\left(\sup _{0<\rho<|z|} T_{q}(\rho, f)(1-|z|)^{\gamma}\right)^{p} d|z|,
$$

for the following values of indexes $\alpha>-1, p, q, s \in(0, \infty), \gamma \geq 0, \tau=(\alpha+1)(p / s)-$ $\gamma p-1$.

The easy proof follows from dyadic decomposition of unit interval and growing properties of $T_{q}(r, f)$ function immediately.

Embeddings from Propositions 2.3-2.5 open a direct way for investigation of properties of less studied analytic area Nevanlinna type spaces via standard and well studied similar type spaces in the unit disk contained for example in [4].

Note Propositions 2.3-2.5 provide valuable information on zero sets of less studied analytic area Nevalinna type spaces based on their connections with standard classes of area Nevanlinna type and the base of proofs of these assertions is again the subharmonicity of $\left(\log ^{+}|f|\right)^{s}$ for $s \geq 1$.

A careful analysis of proofs of emebddings of Ortega and Fabrega, (see [15], [16]), shows us that the following lemma is valid (even in unit ball). The proof is based on subharmonicity of $\left(\log ^{+}|f|\right)^{s}, s \geq 1$.

Lemma 2.1. $L\left(F_{s}^{p, q}\right)(\mathbf{D})$ for all $p \in(1, \infty)$ is a growing function by parameter $q \in$ $(1, \infty]$. This means if $f \in L\left(F_{s}^{p, q_{1}}\right)(\mathbf{D})$ then $f \in L\left(F_{s}^{p, q_{2}}\right)(\mathbf{D})$ if only $q_{1}<q_{2}$.

If $f \in L\left(F_{s q-1}^{p, q}\right)(\mathbf{D})$ then $f \in L\left(F_{t m-1}^{r, m}\right)(\mathbf{D})$, where $m>1, q>1, m<p<r \leq \infty$, $s-1 / p=t-1 / r$.

If $f \in L\left(F_{s q-1}^{p, q}\right)(\mathbf{D})$ then $f \in L\left(A_{t r-1}^{r, p}\right)(\mathbf{D})$, where $p \leq r, q>1, p>1, s-1 / p=$ $t-1 / r$.

\section{On zero sets of area NeVAnlinna type SPACES in Bounded $G$ DOMAins}

Let $G$ be simply connected region on $\mathbb{C}, \partial G$ it is boundary. As usual, $H(G)$ is the space of all analytic functions on $G, \ln ^{+} x=\max _{x>0}(\ln x, 0)$ and also, as usual, $\varphi$ is conformal map of $\mathbf{D}$ on $G, \psi$ is the reverse map and $d(w, \partial G)$ is a distance from $w$ to $\partial G$.

Let $G$ be bounded region, $G \subset \mathbb{C}$ and

$$
N_{\alpha}^{p}(G)=\left\{f \in H(G): \int_{G} d^{\alpha}(w, \partial G)\left(\ln ^{+}|f(w)|\right)^{p} d m_{2}(w)<\infty\right\} .
$$

Definition 3.1. Let $G_{\beta}$ be bounded simply connected region of complex plane. $G_{\beta}$ is $\left(B_{n}\right)$ region if $\partial G_{\beta}$ is equal to $\bigcup_{s=1}^{m} \Gamma_{s}, m \in \mathbb{N}$, where $\Gamma_{s}$ is a smooth arc for which the "angle points" (if they exist) are equal to $\left(\frac{\pi}{\beta_{j}}\right), j=1, \ldots, m$. 
We assume here and below $\beta_{j}=\beta$ for all $j$.

Theorem 3.1. [13] Let $f \in N_{\alpha}^{1}\left(G_{\beta}\right), \frac{1}{2}<\beta \leq 1, f\left(w_{k}\right)=0, f(w) \neq 0, w \neq w_{k}$, $k=1, \ldots$ Then

$$
\sum_{k=1}^{\infty}\left(1-\left|\psi\left(w_{k}\right)\right|\right)^{\frac{\alpha+2}{\beta}}<+\infty .
$$

And if $\left\{w_{k}\right\}_{k=1}^{\infty} \in G_{\beta}$ so that (3.1) holds, then we can construct a function $f \in N_{\alpha}^{1}\left(G_{\beta}\right)$ so that $Z(f)=\left\{w_{k}\right\}_{k=1}^{\infty}$.

Our assertion is the following. We extend partially theorem above to all $0<p \leq 1$.

Theorem 3.2. Let $f \in N_{\alpha}^{p}\left(G_{\beta}\right), 0<p \leq 1, \frac{1}{2}<\beta \leq 1$. Let $f\left(w_{k}\right)=0, f(w) \neq 0$, $w \neq w_{k}, k=1, \ldots$ Then we have

$$
\sum_{k=1}^{\infty}\left(1-\left|\psi\left(w_{k}\right)\right|\right)^{\gamma}<+\infty, \gamma=\frac{\widetilde{\alpha}+2}{p}, \widetilde{\alpha}=\frac{\alpha+2}{\beta}-2,
$$

if $\left(\log ^{+}|f(\psi(z))|\right)^{p}$ is subharmonic for $p \leq 1$ where $\varphi$ is a conformal map from $\mathbf{D}$ on $G_{\beta}$ and $\varphi^{-1}=\psi$.

Remark 3.1. Note also following [13] we can show there is $\tau>0$, so if $\sum_{k=1}^{\infty}(1-$ $\left.\left|\psi\left(w_{k}\right)\right|\right)^{\tau}<+\infty$ there is infinite product $\Pi_{p}\left(z,\left\{\psi\left(w_{k}\right)\right\}\right)=\Pi_{p}\left(z,\left\{z_{k}\right\}\right), p=p(\tau)$, so that $\Pi_{p}\left(z_{k},\left\{z_{k}\right\}\right)=0$ and $\int_{G_{\beta}} d^{\alpha}\left(w, \partial G_{\beta}\right)\left(\ln ^{+}\left|\Pi_{p}(w)\right|\right)^{p}$ is finite.

Proof of Theorem 3.2. The proof is based on (2.3), (2.5) and arguments used in proof of Theorem 3.1. Let $f \in N_{\alpha}^{p}\left(G_{\beta}\right)$. So we have

$$
\|f\|^{p}=\int_{G_{\beta}} d^{\alpha}\left(w, \partial G_{\beta}\right)\left(\ln ^{+}|f(w)|\right)^{p} d m_{2}(w)<+\infty, 0<p \leq 1 .
$$

It is known [17] that $\left|\varphi^{\prime}(z)\right| \approx \frac{d(\varphi(z), \partial G)}{1-|z|}$ and $\frac{1}{\left|\psi^{\prime}(w)\right|} \approx\left(\frac{d(w, \partial G)}{1-|\psi(w)|}\right)$.

Using this we have $(w=\varphi(z))$

$$
\begin{aligned}
\|f\|_{G_{\beta}}^{p} & =\int_{\mathbf{D}}\left(d^{\alpha}\left(\varphi(z), \partial G_{\beta}\right)\right)\left(\ln ^{+}|f(\varphi(z))|\right)^{p}\left|\varphi^{\prime}(z)\right|^{2} d m_{2}(z) \\
& \approx \int_{\mathbf{D}}\left((1-|z|)^{\alpha}\right)\left(\ln ^{+}|f(\varphi(z))|\right)^{p}\left(\left|\varphi^{\prime}(z)\right|^{\alpha+2}\right) d m_{2}(z) \\
& =S
\end{aligned}
$$

It is known for $G_{\beta}$ region [17] that $\left|\varphi^{\prime}(z)\right| \asymp|i-z|^{1 / \beta}$ and $\left|\varphi^{\prime}(z)\right| \asymp|i-z|^{1 / \beta-1}$, hence

$$
S \asymp \int_{\mathbf{D}}(1-|z|)^{\alpha}\left(\ln ^{+}|f(\varphi(z))|\right)^{p}|i-z|^{(1 / \beta-1)(\alpha+2)} d m_{2}(z)
$$

but $|i-z|^{1 / \beta-1} \geq(1-|z|)^{(1 / \beta-1)}, \beta \in(0,1]$. So finally

$$
\left.\|f\|_{G_{\beta}}^{p} \geq C\left(\int_{\mathbf{D}}(1-|z|)^{\frac{\alpha+2}{\beta}-2}\right)\left(\ln ^{+}|f(\varphi(z))|\right)^{p} d m_{2}(z)\right) .
$$


Put $F(z)=f(\varphi(z))$, it remains to use (2.3), for $0<p \leq 1, F \in H(D)$ and estimate $(2.5)$.

Theorem 3.2 is proved.

Remark 3.2. It will be interesting to know is the last assertion of this paper sharp.

Acknowledgment: Second author is supported by MNTR Serbia, Project 174017.

\section{REFERENCES}

[1] M. Arsenović, R. F. Shamoyan, On zero sets and parametric representations of some new analytic and meromorphic function spaces in the unit disk, Filomat, 25(3), (2011), 1-14.

[2] L. Carleson, Interpolations by bounded analytic functions and the corona problem, Annals of Mathematics, 76(3), (1962), 547-559.

[3] B. R. Choe, H. Koo, W. Smith, Carleson measures for the area Nevanlinna spaces and applications, Journal d'Analyse mathématique, vol 104 (2008), 207-229.

[4] M. M. Djrbashian, F. A. Shamoian, Topics in the Theory of $A_{\alpha}^{p}$ Spaces, Leipzig, Teubner, 1988.

[5] T. M. Flett, Inequalities for the p-th mean values of harmonic and subharmonic functions with $p \leq 1$,, Proc. London Math. Soc. (3), 20 (1970), 249-275.

[6] W. W. Hastings, A Carleson measure theorem for Bergman spaces, Proc. Amer. Math. Soc. 52 (1975), 237-241.

[7] W. Hayman, Meromorphic functions, Oxford University Press, 1964.

[8] H. Hedenmalm, B. Korenblum, K. Zhu, Theory of Bergman spaces, Springer-Verlag, New York, 2000.

[9] H. Jarchow, J. Xiao, Composition operators between Nevanlinna classes and Bergman spaces with weights, J. Operator Theory, 46 (2001), no. 3, suppl., 605-618.

[10] M. Jevtić, M. Pavlović, R. Shamoyan, A note on diagonal mapping theorem in spaces of analytic functions in the unit polydisk, Publ. Math. Debrecen, 74/1-2, 2009, 1-14.

[11] V. L. Oleinik, Embeddings theorems for weighted classes of harmonic and analytic functions, J. Soviet Math. 9 (1978), 228-243.

[12] V. L. Oleinik, B. S. Pavlov, Embedding theorems for weighted classes of harmonic and analytic functions, J. Soviet Math. 2 (1974), 135-142.

[13] O. Prichodko, On description of zero sets of some analytic spaces of area Nevanlinna type in angular domain, Vesntik Bryansk State University, No. 4, 2010.

[14] F. A. Shamoyan, Parametric representation and description of the root sets of weighted classes of functions holomorphic in the disk, Siberian Math. Journal, 40(6) (1999), 1211-1229.

[15] J. Ortega, J. Fabrega, Hardys inequality and embeddings in holomorphic Lizorkin-Triebel spaces, Illinois Journal of Mathematics, Vol. 43 4, (1999), 733-751.

[16] J. Ortega, J. Fabrega, Holomoprhic Triebel-Lizorkin spaces, Journal of functional Analysis, Vol. 151, (1997), 177-212.

[17] F. A. Shamoyan, V. Bednag, O. Prichodko, On zero sets of some weighted classes of analytic functions, in Russian, Vestnik Bryansk State University, No. 4, 2008.

[18] F. A. Shamoyan, E. N. Shubabko, Parametric representations of some classes of holomorphic functions in the disk, Complex analysis, operators and related topics Operator theory Adv. applications, Basel, 113 (2000), 331-338.

[19] R. F. Shamoyan, On multipliers from Bergman type spaces into Hardy spaces in the polydisc, in Russian, Ukrainian. Math. Journal, No. 10, (2000), 1405-1415.

[20] R. F. Shamoyan, H. Li, Description of zero sets of certain analytic spaces of area Nevanlinna type, Kragujevac Math. Journal, 34 (2010), 73-89. 
[21] R. F. Shamoyan, O. Mihić, On some new theorems on certain analytic and meromorphic classes of Nevanlinna type on the complex plane, Kragujevac Journal of Mathematics, Volume 37(1), (2013), 65-85.

\author{
${ }^{1}$ BRYANSK UNIVERSITY, \\ RUSSIA \\ E-mail address: rshamoyan@yahoo.com \\ ${ }^{2}$ Faculty of Organisational Sciences \\ UNIVERSITY OF BELGRADE, \\ SERBIA \\ E-mail address: oliveradj@fon.rs
}

